\title{
Inter-symbol interference and silicon photomultiplier VLC receivers in ambient light.
}

\author{
William Matthews, Wajahat Ali, Zubair Ahmed, Grahame Faulkner and Steve Collins Member, IEEE
}

\begin{abstract}
Results of an investigation into the performance of a wide field of view silicon photomultiplier (SiPM) receiver under ambient light are reported. During these investigations a new form of inter-symbol interference (ISI) was discovered, which can be attributed to saturation of the SiPM. This new form of ISI means that using decision feedback equalization (DFE) improves the receiver's performance at low data rates.
\end{abstract}

Index Terms-Visible Light Communications, Optical Wireless Communications, Silicon Photomultiplier, Intersymbol Interference

\section{INTRODUCTION}

$\mathrm{T}$ he capacity of a visible light communication (VLC) or optical wireless communication (OWC) channel depends upon the channel's bandwidth and the receiver's signal to noise ratio (SNR). The statistical variations in the number of photons incident on a receiver in a symbol time means that Poisson noise is the lowest possible noise level in any receiver and this noise level is achieved by counting individual photons. In the past few years, the advent of single photon avalanche diodes (SPADs) has allowed VLC receivers to be created which can count photons [1-3].

A SPAD is made by placing an avalanche photodiode (APD) in series with a quenching device and biasing it above its breakdown voltage. However, the resulting SPAD needs several nanoseconds to recharge after detecting a photon. The problems associated with the resulting recovery time mean that silicon photomultipliers (SiPMs), which are arrays of SPADs [1,2], are preferred in receivers. Recently, a SiPM receiver has been shown to operate within a factor 1.7 of the Poisson limit at a data rate of 1 Gbps [4]. Subsequently, another SiPM has achieved a maximum data rate of $3.45 \mathrm{Gbps}$ [5]. However, these results were obtained when a Thorlabs FB405-10 optical bandpass filter, with a central wavelength of $405 \mathrm{~nm}$ and a FWHM of $10 \mathrm{~nm}$, was used to reject most of the ambient light. Unfortunately, this filter restricts the field of

This manuscript was submitted to IEE Photonics Technology Letters on November $23^{\text {rd }}, 2020$. This work has been partly supported by the Punjab Educational Endowment Fund, Pakistan and by the UK Engineering and Physical Sciences Research Council (EPSRC) under Grant EP/R00689X/1.W.Matthews (william.matthews@eng.ox.ac.uk), W.Ali (wajahat.ali@eng.ox.ac.uk), Z.Ahmed (zubair.ahmed@eng.ox.ac.uk), G.Faulkner (grahame.faulkner@eng.ox.ac.uk) and S.Collins (steve.collins@eng.ox.ac.uk) are with the Engineering Science Department, University of Oxford, Oxford, UK.
TABLE I

KEY PARAMETERS OBTAINED FROM THE MANUFACTURERS DATA SHEET FOR A J SERIES 30020 OPERATING WITH AN OVERVOLTAGE OF $5 \mathrm{~V}[6]$.

\begin{tabular}{|c|c|}
\hline Parameter & Value \\
\hline Area $\left(\mathrm{mm}^{2}\right)$ & 9.42 \\
\hline Number of Microcells & 14410 \\
\hline Microcell active area diameter $(\mu \mathrm{m})$ & 20 \\
\hline Fill Factor $(\%)$ & 62 \\
\hline Recovery Time $(\mathrm{ns})$ & $15 \mathrm{~ns}$ \\
\hline PDE (405 nm) & 0.38 \\
\hline Dark Count Rate $(\mathrm{MHz})$ & 1.18 \\
\hline Pulse Width $(\mathrm{ns})$ & 1.4 \\
\hline
\end{tabular}

view $(\mathrm{FoV})$ of the receiver.

In this paper the results of an investigation into the impact of removing this optical filter are reported. One consequence of removing the filter is a previously unreported form of intersymbol interference (ISI), that can be attributed to the saturated response of the SiPM. The most important consequence of removing the filter is that decision feedback equalization (DFE) can have a significant impact on the receiver performance at low data rates.

This paper is organized as follows. The operation and characteristics of SiPMs are discussed in section II. The experimental equipment used to test SiPMs as receivers and the results of experiments are described in sections III and IV. Finally, section V contains concluding remarks.

\section{SIPM CHARACTERISTICS}

SiPMs are now manufactured by companies, including Hamamatsu, Ketek and ON-Semiconductor. In these devices each detected photon creates an output pulse that can be counted. Hence SiPMs have the potential to form the basis for the most sensitive possible VLC receivers.

When selecting a SiPM for use in a VLC receiver an important consideration is the inter-symbol interference (ISI) that might be caused by its output pulses. This means that an important characteristic when selecting a SiPM for a VLC receiver is the duration of its output pulses. ONSemiconductor manufactures SiPMs with two outputs. Slower pulses on one output arise from the recharging of a microcell after a photon has been detected. Faster output pulses are then created by capacitive coupling a second output to each microcell. For the $\mathbf{J}$ series SiPM, whose parameters are listed in Table I, the full-width at half maximum of these faster pulses is $1.4 \mathrm{~ns}$ [6]. This means that ISI is expected to occur 


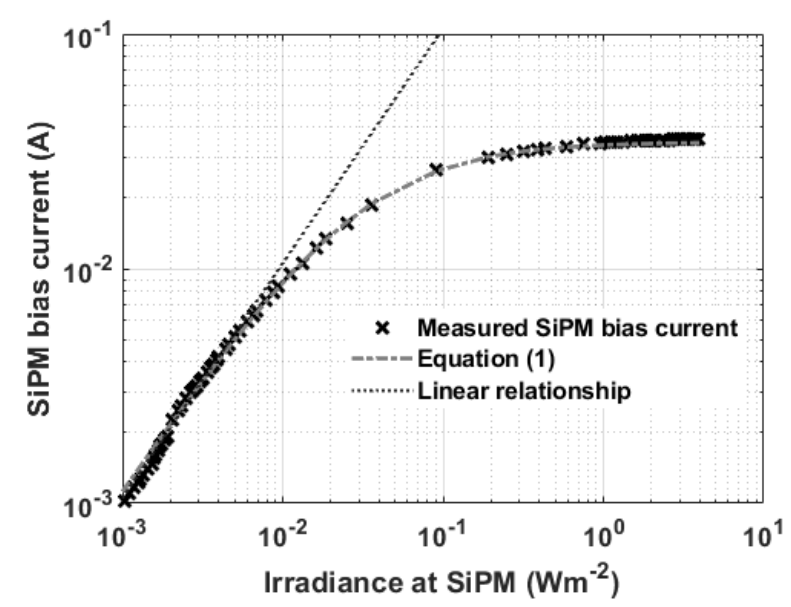

Fig. 1. The variation of the bias current of a J-30020 SiPM with irradiance from a $405 \mathrm{~nm}$ laser diode when the applied bias is $27.5 \mathrm{~V}$. The measured data is compared to a linear relation at lower irradiances and equation (1) for all irradiances.

when the OOK data rate is more than $500 \mathrm{Mbps}$.

Another important feature of SiPMs is that each microcell must be quenched after a photon is detected. This is achieved by reducing the bias voltage across the active area of the microcell to less than its breakdown voltage. The bias voltage then needs a finite time to recharge. This recharge or recovery time is therefore an important characteristic of a SiPM.

The recovery time listed in Table I was measured when the SiPM was connected to a $1 \Omega$ load resistance. However, the SiPM used to create a receiver was mounted on a MicroFJ30020 evaluation board that includes a $50 \Omega$ load resistance. The recovery time of the SiPM on the board was therefore determined by measuring the duration of individual slow output pulses. These pulses showed the expected exponential decay with a characteristic time, $\tau$, of $30 \mathrm{~ns}$, which corresponds to a recharge time of $66 \mathrm{~ns}$. When the irradiance on the SiPM increases, the rate at which microcells discharge and hence the current required to maintain the SiPM bias voltage also increases. However, the finite recovery time means that, as shown in Fig. 1, the rate at which photons are detected, and hence this current, saturates.

Starting from a relationship between count rate and irradiance [7], the bias current needed to recharge the microcells when an irradiance $L$ is falling on a SiPM is

$$
I_{\text {bias }}=\frac{Q_{\text {cell }} N_{\text {cells }} \alpha\left(L+L_{\text {dark }}\right)}{1+\alpha \tau_{\text {recharge }}\left(L+L_{\text {dark }}\right)}
$$

In this expression $Q_{\text {cell, }} N_{\text {cells, }} \tau_{\text {recharge }}$ and $L_{\text {dark }}$ are the charge needed to recharge a microcell, the number of microcells, the recharge time and the irradiance that is equivalent to the dark count rate of the SiPM. In addition, if $\eta$ is the photon detection efficiency (PDE) of the SiPM at the wavelength of the irradiating light, $A_{\mathrm{SiPM}}$ is the SiPM area and $E_{p}$ is the photon energy then

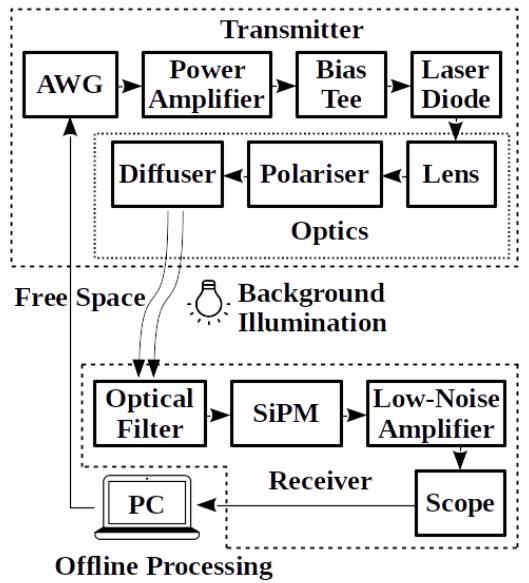

Fig. 2. System block diagram describes the experimental setup used to evaluate the performance of the selected SiPM.

$$
\alpha=\frac{\eta A_{\mathrm{SiPM}}}{E_{p}}
$$

The one parameter in (1) that is unknown is the charge stored on each microcell. The bias current and photon count rate have therefore been measured in the dark. Their ratio shows that the charge needed to recharge a microcell is $0.22 \mathrm{pC}$. Furthermore, the bias current in the dark is only $0.26 \mu \mathrm{A}$. This means that $L_{\text {dark }}$ has an insignificant impact on the results in Fig 1. Consequently, for the lower irradiances in Fig 1 the SiPM bias current is proportional to the irradiance falling on the SiPM. More importantly, with an effective PDE of $38 \%$ (1) agrees with the measured data over a wide range of irradiances. A comparison of the measured response and the extrapolated linear region shows that the linear region ends when the bias current is approximately $10 \mathrm{~mA}$. The bias current then saturates to a maximum of approximately $40 \mathrm{~mA}$.

\section{EXPERIMENT SET-UP}

The narrow pass-band optical filter used in previous experiments $[4,5]$ restricts the receiver's FoV. The impact of removing this optical filter from the receiver has therefore

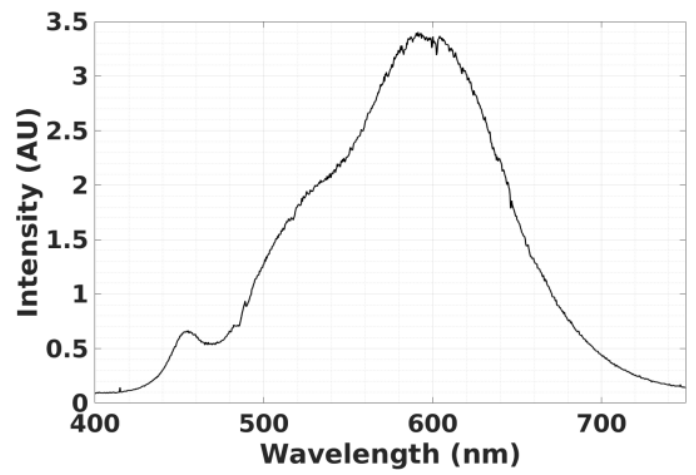

Fig. 3. White LED spectrum from the 8 W Philips IBRS 10461 domestic lighting bulb used as background illumination. 
been investigated using the equipment shown in Fig. 2. In this experimental set-up, a $10 \mathrm{GHz}$ Tektronix Arbitrary Waveform Generator (AWG) generated a pseudorandom binary sequence (PRBS). The output of this AWG was then amplified by a Mini-Circuits ZFL-1000H+, $10 \mathrm{MHz}$ to $1 \mathrm{GHz}$, amplifier. The resulting signal was then added to a DC bias voltage by a Mini-Circuits Bias-Tee (ZFBT-4R2GW+). The output from the Bias-Tee was applied to a $405 \mathrm{~nm}$ laser diode (L405P20). The final part of the transmitter was a polarizer that was used to vary the transmitted irradiance falling on the SiPM.

The SiPM was also illuminated by a warm white LED $(8 \mathrm{~W}$ Philips IBRS 10461), whose spectrum is shown in Fig. 3. To replicate the worst-case conditions which might occur in an office the light from the LED was directed towards the SiPM so that it provided 500 lux to the surface of the SiPM

The receiver consisted of a SiPM mounted on a MicroFJ30020 evaluation board whose fast output was connected to a ZX6043-S+ 4GHz amplifier and then to a Keysight MSOV334A 33GHz, $80 \mathrm{GSa} / \mathrm{s}$ oscilloscope. The received $8 \mathrm{~b} 10 \mathrm{~b}$ data was then post-processed in MATLAB ${ }^{\circledR}$. The first processing step was a $4^{\text {th }}$ order Butterworth low pass filter, with a pass-band proportional to the data rate. If required, decision feedback equalization (DFE) was applied to the filtered signal. Finally, the bit error rate (BER) was calculated.

\section{EXPERIMENTAL RESULTS}

With the experimental setup described in section III excellent results have previously been obtained when a FB405-10 optical bandpass filter was incorporated into the receiver $[4,5]$. The simplest way to increase the receiver fieldof-view is to remove this filter. Experiments were therefore performed in 500 lux of ambient light from the warm white LED with and without a FB405-10 filter in place. Some experiments were performed at data rates, such as $100 \mathrm{Mbps}$, at which ISI caused by the finite width of the SiPM pulses is expected to have little effect. However, experiments were performed both with DFE and without DFE for all data rates. In each experiment the transmitter was $30 \mathrm{~cm}$ above the SiPM. The polarizer was then used to vary the irradiance from the

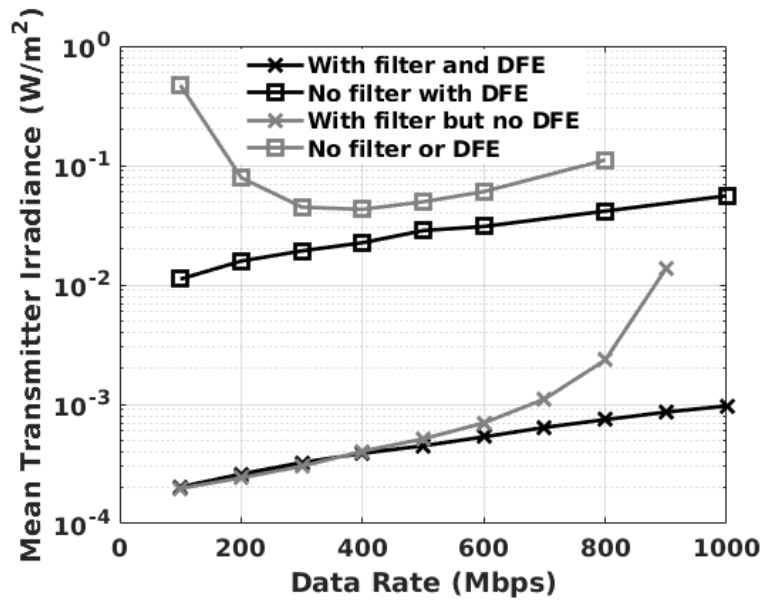

Fig. 4. The irradiance required to achieve a BER of $10^{-3}$ when the 405 $\mathrm{nm}$ filter is in place and after it was removed. In both cased experiments were performed without DFE and with DFE. transmitter falling on the SiPM until a particular BER was achieved. The existence of a range of forward error correction codes [8] and desirable BERs after correction means that the performance of VLC systems have previously been reported at BERs of $10^{-3}[9], 2 \times 10^{-3}[3]$ and $3.8 \times 10^{-3}[10,11]$. Since lower BERs are more difficult to achieve the results in Fig 4 were obtained with a BER of $10^{-3}$. With DFE it was possible to obtain this BER at a data rate of 1 Gbps. However, without DFE the maximum data rate that could be achieved with the maximum available irradiance was lower. The results in Fig 4 show that, as expected, with the filter in place applying DFE only has an impact on data rates of $500 \mathrm{Mbps}$ or higher. However, when the receiver is operated without a filter, applying DFE has a larger impact on data rates less than 400 Mbps.
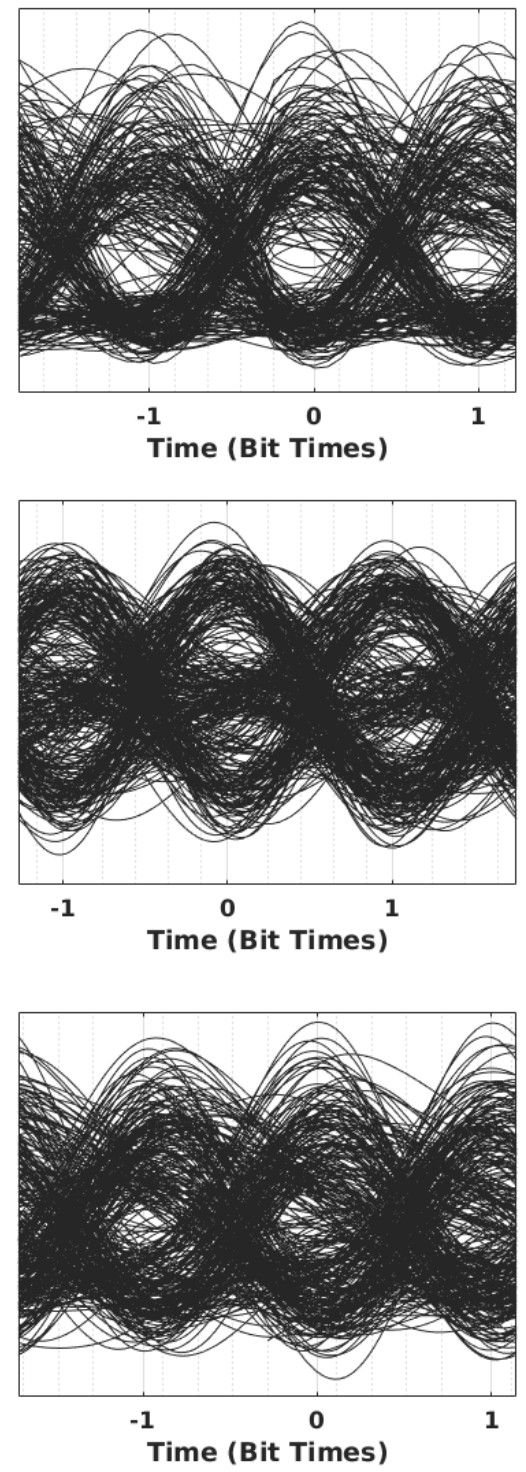

Fig. 5. Eye diagrams before DFE is applied at $100 \mathrm{Mbps}$ in 500 lux of ambient light from a warm LED with a FB405-10 filter (top), with 500 lux of ambient light without a filter in place (middle) and when the ambient light level has been reduced to give $20.5 \mathrm{~mA}$ of photocurrent (bottom). In all cases the BER after DFE was applied was $10^{-3}$. 
The cause of the unexpected benefits of applying DFE at low data rates has been investigated by comparing eye diagrams. The eye diagrams before DFE is applied at $100 \mathrm{Mbps}$ in 500 lux of ambient light with the optical filter in place and after it has been removed are shown in Fig. 5. A comparison of these two eye diagrams shows that when the filter is removed a third level appears in the eye diagram. Another significant change when the filter is removed from the receiver is that the current needed to maintain the SiPM bias voltage increases from $0.27 \mathrm{~mA}$ to $31 \mathrm{~mA}$. A comparison of these currents with the results in Fig. 1 shows that with the filter in place the SiPM is operating in its linear region. However, with the filter removed the SiPM's response is close to saturation. This suggests that the new level in the eye diagram is caused by the saturation of the SiPM. To investigate this possibility the ambient light reaching the receiver was reduced until the bias current was $20.5 \mathrm{~mA}$, which corresponds to 62 lux. Fig. 1 shows that at this bias current the SiPM's response is non-linear but not yet approaching saturation. As shown at the bottom of Fig. 5 under these conditions the additional third level is absent from the eye diagram. This extra level in the eye diagram is therefore associated with the saturation of the SiPM.

Saturation is caused by a reduction in the effective PDE arising from the increasing number of microcells recovering after detecting a photon at higher irradiances. When data is transmitted to a SiPM near saturation the effective PDE during a symbol time then depends upon the preceding bits. At $400 \mathrm{Mbps}$ the bit time, $2.5 \mathrm{~ns}$, means that there are 26 bits during the J-30020's 66 ns recovery time. Consequently, with $8 \mathrm{~b} 10 \mathrm{~b}$ coding, each bit suffers from a similar reduction in PDE. In contrast, at $100 \mathrm{Mbps}$ there are fewer than 7 bits in the recovery time and the reduction in PDE will vary between bits. This data rate dependence of the variability of the effective PDE for different bits possibly explains why DFE has a larger impact at data rates less than $400 \mathrm{Mbps}$ in Fig (4).

The most important conclusion from Fig. 4 is that conventional DFE has a significant impact on this new form ISI. Despite the benefits of DFE the results in Fig. 4 show that in ambient light removing the filter increases the irradiance required to support data rates between $100 \mathrm{Mbps}$ and $1 \mathrm{Gbps}$ by a factor of approximately 60 . Removing the filter therefore increases the receiver's field of view but at the cost of significantly increasing the irradiance required to transmit data. Consequently, without a filter this SiPM requires an average transmitter irradiance at the receiver of $55.7 \mathrm{~mW} / \mathrm{m}^{2}$ to support $1 \mathrm{Gbps}$ with a BER of $10^{-3}$. However, the spectrum of the white LED in Fig. 3 shows that only a small fraction of the ambient light is at wavelengths close to $405 \mathrm{~nm}$. A suitable wide field of view filter is therefore expected to improve the receiver's performance. The challenge is to find a wide field of view filter that blocks the ambient light without significantly attenuating the signal from the transmitter.

\section{CONCLUSION}

Previously, results have been reported when a narrow optical band-pass filter and a SiPM have been used to create a VLC receiver operating in ambient light [4,5]. However, this optical filter limits the receiver's field of view.
The results of experiments to determine the impact of removing the optical band-pass filter have been reported for the first time. An unexpected consequence of removing the filter was that DFE had a significant impact at data rates less than $400 \mathrm{Mbps}$. The eye diagrams before and after the filter was removed showed that removing the filter introduces a third level into the eye diagram. A comparison of three eye diagrams showed that this previously unreported form of ISI arises because the ambient light forces the SiPM to operate close to saturation.

In the future, with a suitable wide field of view optical filter, lower irradiances than those in Fig. 4 will be required to support $1 \mathrm{Gbps}$. Alternatively, higher OOK data rates will be supported with the same irradiance. In fact, when operating in ambient light from a white LED a filter may prevent the occurrence of this new form of ISI. However, the higher irradiance of outdoor daylight may mean that, even with a filter in place, DFE could still be beneficial at low data rates when operating outdoors. In addition, the high peak powers associated with orthogonal frequency-division multiplexing (OFDM) means that this new form of ISI might be caused by the OFDM signal itself. In the future, a scheme will therefore be required to prevent this new form of ISI from limiting the OFDM data rate that can be transmitted to a SiPM.

\section{REFERENCES}

[1] E. Fisher, I. Underwood, and R. Henderson, "A reconfigurable 14-bit 60Gphoton/s Single-Photon receiver for visible light communications," Eur. Solid-State Circuits Conf., pp. 85-88, 2012.

[2] D. Chitnis and S. Collins, "A SPAD-based photon detecting system for optical communications," J. Light. Technol., vol. 32, no. 10, pp. 2028-2034, 2014.

[3] H. Zimmermann, "APD and SPAD Receivers: Invited Paper," 2019 15th International Conference on Telecommunications (ConTEL), Graz, Austria, 2019, pp. 1-5, doi: 10.1109/ConTEL.2019.8848547.

[4] Z. Ahmed, R. Singh, W. Ali, G. Faulkner, D. O'Brien and S. Collins, "A SiPM-Based VLC Receiver for Gigabit Communication Using OOK Modulation," in IEEE Photonics Technology Letters, vol. 32, no. 6 , pp. $317-320$

[5] W. Matthews, Z. Ahmed, W. Ali and S. Collins, "A SiPM-based VLC Receiver for 3.45 Gigabits/s Communication Using OOK Modulation," 2020 IEEE Photonics Conference (IPC), Vancouver, BC, Canada, 2020, pp. 1-2

[6] Onsemi.com. 2020. J-Series SiPM Sensors Datasheet. [online] Available at: https://www.onsemi.com/pub/Collateral/MICROJSERIES-D.PDF, Accessed 10 March 2020.

[7] Zhang, L., et al. "A Comparison of APD and SPAD Based Receivers for Visible Light Communications." Journal of Lightwave Technology, vol. 36, no. 12, Journal of Lightwave Technology, 2018, pp. $2435-42$

[8] "Forward error correction for high bit rate DWDM submarine systems," Int. Telecommun. Union, Geneva, Switzerland, ITURecommendation G. 975.1, Feb. 2004.

[9] S. Huang and M. Safari, "Hybrid SPAD/PD Receiver for Reliable Free-Space Optical Communication," in IEEE Open Journal of the Communications Society, vol. 1, pp. 1364-1373, 2020, doi: 10.1109/OJCOMS.2020.3023009.

[10] Yuanquan Wang, Yiguang Wang, Nan Chi, Jianjun Yu, and Huiliang Shang, "Demonstration of 575-Mb/s downlink and 225-Mb/s uplink bi-directional SCM-WDM visible light communication using RGB LED and phosphor-based LED," Opt. Express 21, 1203-1208 (2013)

[11] R. Bian, I. Tavakkolnia and H. Haas, "15.73 Gb/s Visible Light Communication With Off-the-Shelf LEDs," in Journal of Lightwave Technology, vol. 37, no. 10, pp. 2418-2424, 15 May15, 2019, doi: 10.1109/JLT.2019.2906464. 